\title{
Comparação da Capacidade de Sistemas Celulares usando a Plataforma J2ME
}

\author{
Michelle H. Araújo e Ivan R. S. Casella \\ Universidade Federal do $A B C$ \\ Centro de Engenharia, Modelagem e Ciências Sociais Aplicadas
}

Resumo- Este artigo apresenta uma aplicação da tecnologia J2ME no desenvolvimento de um programa, que pode ser executado em dispositivos móveis como celulares e handhelds, para o cálculo da capacidade das principais tecnologias de sistemas celulares existentes na atualidade.

Palavras-chave - J2ME, Sistemas Celulares.

\section{Introdução}

Os sistemas de comunicação pessoal (PCS) têm sofrido continuamente grandes modificações para atender a crescente demanda por novos serviços e aplicações de alta velocidade com capacidade multimídia. Devido às grandes vantagens de portabilidade dos aparelhos de comunicação móveis e a crescente necessidade de aplicativos cada vez mais sofisticados com recursos multimídia, foi desenvolvida pela Sun Microsystems uma linguagem especialmente dedicada a dispositivos portáteis com diferentes capacidades de processamento e com recursos limitados, o J2ME.

Neste contexto, nesse artigo são apresentados dois aplicativos desenvolvidos em J2ME para o cálculo da capacidade, em canais por célula e em Erlang, para sistemas que utilizam as tecnologias FDMA (Frequency Division Multiple Access), TDMA (Time Division Multiple Access) e CDMA (Code Division Multiple Access). O cálculo da capacidade em Erlang foi acrescentado aos aplicativos já que ele oferece uma informação estatística adicional para um dimensionamento mais realista dos sistemas celulares. O primeiro aplicativo utiliza a configuração CLDC (Connected Limited Device configuration) e perfil MIDP 2.1 (Mobile Information Device Profile), enquanto o segundo foi desenvolvido com a configuração CDC (Connected Device Configuration). Ambos os aplicativos são adequados para os atuais dispositivos portáteis encontrados no mercado e levam em consideração as limitações de processamento existentes.

\section{Cálculos}

Os aplicativos foram desenvolvidos levando em consideração algumas fórmulas de capacidade bastante conhecidas na literatura científica [1].

Para o cálculo da capacidade, dada em número de canais por célula, para sistemas FDMA (e.g. AMPS [1]), tem-se:

$N_{c}=\frac{W_{F D M A} \cdot 10^{3}}{B_{c}} \cdot \frac{1}{f_{\text {reuso }}} \cdot f_{\text {ho }}$

Para o cálculo da capacidade, dada em número de canais por célula, para sistemas TDMA (e.g. IS-136 e GSM [1]), tem-se:

$N_{c}=\frac{W_{T D M A} \cdot 10^{3}}{B_{c}} \cdot \frac{1}{f_{\text {reuso }}} \cdot f_{\text {ho }} \cdot f_{\text {mux }}$
Para o cálculo da capacidade, dada em número de canais por célula, para sistemas CDMA (e.g. IS-95 [1]), tem-se:

$$
N_{c}=\frac{W_{C D M A} \cdot 10^{3}}{R_{b} \cdot\left(E_{b} / N_{o}\right)} \cdot \frac{1}{f_{v o z}} \cdot \frac{1}{\left(1+f_{\text {int }}\right)} \cdot f_{h o} \cdot f_{p c} \cdot f_{\text {ant }} \cdot N_{\text {port }}
$$

Onde,

$N_{c}$ é o número de canais disponível no sistema

$W_{F D M A}$ é a largura de faixa ocupada pelo sistema FDMA em $\mathrm{MHz}$

$W_{T D M A}$ é a largura de faixa ocupada pelo sistema TDMA em $\mathrm{MHz}$

$W_{C D M A}$ é a largura de faixa ocupada pelo sistema CDMA em $\mathrm{MHz}$

$B_{c}$ é a banda ocupada por um canal FDMA ou TDMA em kHz

$f_{\text {reuso }}$ é o fator de reuso de canal

$f_{\text {mux }}$ é o fator de ganho devido à multiplexação do canal no tempo

$f_{h o}$ é o fator perda por hand-off

$R_{b}$ é a taxa de bits transmitida em kbps

$\left(E_{b} / N_{o}\right)$ é a relação entre energia de bit e densidade espectral de ruído $f_{v o z}$ é o fator de utilização da voz

$f_{\text {int }}$ é o fator de interferência vinda de outras células do sistema $f_{p c}$ é o fator de perda devido a imprecisão do controle de potência $f_{\text {ant }}$ é o fator de ganho devido a setorização (antenas direcionais) $N_{\text {port }}$ é o número de portadoras disponíveis

Nesse trabalho, será considerado que as células são divididas em 3 setores. Deste modo, o cálculo da capacidade em número de canais por setor é obtido pela divisão do número de canais por célula por 3 .

Para o cálculo da capacidade baseada no tráfego em Erlang, é utilizado um algoritmo iterativo baseado em (4) para determinar a densidade de tráfego total do setor $\left(A_{s}\right)$ [1].

$P_{b}=\frac{A_{s}^{N} / N !}{\sum_{i=0}^{N} A_{s}^{i} / i !}$

Onde,

$P_{b}$ é a probabilidade de todos os canais estarem ocupados

$N$ é a quantidade de canais por setor

Considerando que o tráfego gerado por um único usuário $\left(A_{\text {user }}\right)$ possa ser determinado por:

$A_{\text {user }}=T_{\text {con }} \cdot N_{\text {con }} / 60$

Onde,

$T_{c o n}$ é a duração de uma conexão em minutos

$N_{c o n}$ é o número de conexões em 1 hora

Pode-se, então, obter a capacidade em número de usuários $\left(N_{u}\right)$ baseada no tráfego em Erlang, fazendo:

$N_{u}=3 \cdot A_{s} / A_{u s e r}$ 


\section{Desenvolvimento dos Aplicativos J2ME}

A flexibilidade do ambiente J2ME possibilita criar aplicativos para dispositivos móveis de acordo com sua capacidade de processamento. Com base nessa característica, foram desenvolvidos dois aplicativos para dispositivos móveis que permitem calcular a capacidade de sistemas celulares.

O primeiro aplicativo foi desenvolvido utilizando a configuração $\mathrm{CDC}$, que é destinada a dispositivos portáteis com uma capacidade maior de processamento (e.g. pocket PC). Nas figuras 1 e 2 são apresentadas as principais telas do aplicativo CDC. Pode-se notar que a apresentação do aplicativo é muito semelhante àquela dos aplicativos para computadores de mesa e que todos os parâmetros podem ser encontrados numa mesma tela. Na figura 1 são apresentados os resultados de capacidade em número de canais e na figura 2 são apresentados os resultados em número de usuários, baseados no tráfego em Erlang, para os sistemas analisados (AMPS, GSM e IS-95). Os parâmetros de cada tecnologia utilizados nos cálculos são apresentados nas figuras.

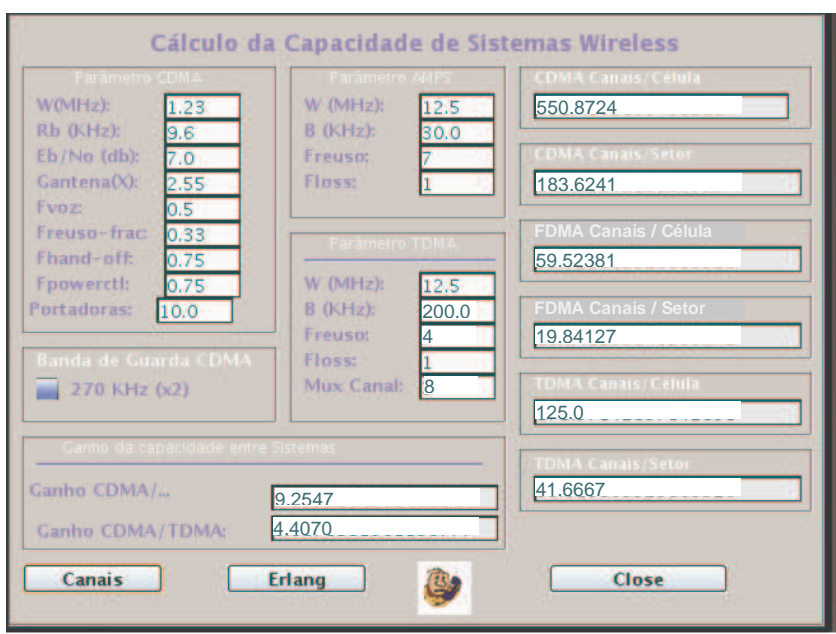

Figura 1- Resultados em número de canais para o aplicativo CDC

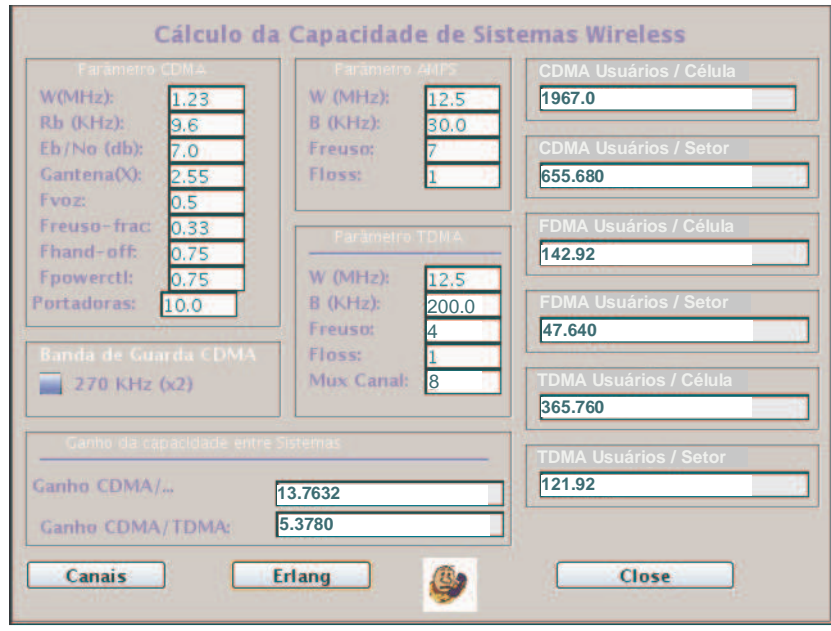

Figura 2- Resultados baseados em Erlang para o aplicativo CDC

O segundo aplicativo foi desenvolvido utilizando a configuração CLDC, que é adequada para dispositivos com uma capacidade limita de processamento (e.g celulares e handhelds). Nas figuras 3 e 4 são apresentadas as principais telas do aplicativo CLDC. Pode-se notar que os parâmetros e resultados foram divididos em mais de uma tela, devido à dimensão reduzida desses dispositivos. Em função das limitações matemáticas do CLDC, foi desenvolvida adicionalmente uma biblioteca matemática para a realização dos cálculos. Os resultados de capacidade para os sistemas analisados (AMPS, GSM e IS-95) utilizando os mesmos parâmetros da configuração $\mathrm{CDC}$, em número de canais e em número de usuários, são apresentados nas figuras 3 e 4.

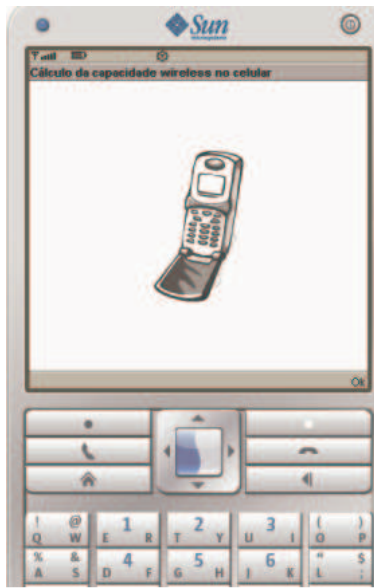

\begin{tabular}{|l||}
\hline Resultados: \\
\hline CDMA Canais/Setor: \\
\hline 183.6241 \\
\hline CDMA Canais/Célula: \\
\hline 550.8724 \\
\hline FDMA Canais/Setor: \\
\hline $19.84127 \quad \vdots$ \\
\hline FDMACanais/Célula: \\
\hline 59.52381 \\
\hline TDMA Canais/Setor: \\
\hline 41.66667 \\
\hline TDMA Canais/Célula: \\
\hline 125.0 \\
\hline Ganho da Capacidade entre Sistemas: \\
\hline Ganho CDMA/AMPS: \\
\hline 9.2547 \\
\hline Ganho CDMA/DMM: \\
\hline 4.4070 \\
\hline Back \\
\hline
\end{tabular}

Figura 3- Resultados em número de canais para o Aplicativo CLDC

\begin{tabular}{|c|c|}
\hline$\varphi_{\text {Inlll }}=0$ & Resultados com a capacidade de Erlang \\
\hline Fórmula de Erlang $\mathrm{B}$ & Número de Úsuários (CDMA): \\
\hline Probabilidade de Blocking (\%): & 1967.0 \\
\hline 11 & Ganho CDMAFDMA em erlang: \\
\hline $\begin{array}{l}\text { Quantidade de chamadas por hora: } \\
5\end{array}$ & 13.7632 \\
\hline $\begin{array}{l}\text { Duração média de cada chamada (em minutos): } \\
3\end{array}$ & $\begin{array}{l}\text { Ganho CDMA/TDMA em erlang: } \\
5.3780\end{array}$ \\
\hline $\begin{array}{l}\text { Múmero de canais(FDMA / Setor): } \\
19.84127\end{array}$ & \\
\hline $\begin{array}{l}\text { Húmero de canais (TDMA / Setor): } \\
41.66667\end{array}$ & \\
\hline $\begin{array}{l}\text { Húmero de canais (CDMA / Setor): } \\
183.6241\end{array}$ & \\
\hline Back & Exit \\
\hline
\end{tabular}

Figura 4- Resultados baseados em Erlang para o aplicativo CLDC

\section{Conclusão}

Nos últimos anos, os dispositivos móveis tem sido utilizados para aplicações cada vez mais sofisticadas e em diversas áreas de atuação. Nesse contexto, foram apresentadas duas implementações possíveis usando as configurações CLDC e CDC do J2ME no desenvolvimento de um aplicativo para o cálculo da capacidade, em número de canais e em número de usuários baseada no tráfego em Erlang, de sistemas celulares empregando diferentes tecnologias. Considerando os parâmetros analisados, pode-se verificar que os sistemas CDMA apresentam um ganho significativo de capacidade tanto em número de canais (9.3 e 4.4), como em número de usuários (13.8 e 5.4), em relação aos sistemas AMPS e GSM.

\section{Referências Bibliográficas}

[1] V. K. Garg, "Wireless Network Evolution: 2G to 3G", Prentice Hall, 2001.

[2] J. W. Muchow, "Core J2ME: Tecnologia \& MIDP", Pearson Markron Books, 2007. 